\title{
Una aproximación teórica al desarrollo endógeno
}

Alfredo Macías ${ }^{1}$ y Gonzalo Saavedra²

\section{RESUMEN}

Este trabajo propone una reflexión teórica y conceptual acerca del desarrollo como proceso endógeno. La viabilidad de tal perspectiva depende, en gran medida, de si se asume o no su construcción a partir de la vida comunitaria como espacio de subjetividad. La tarea es compleja pero posible, pues parece depender de las propias comunidades y de sus capacidades para sobreponer sus facultades reflexivas a un ethos racionalista, propio de una civilización que progresivamente niega y desestima sus dimensiones morales y afectivas. Es necesario entonces superar el fatalismo determinista de lo estructural, el reto del desarrollo endógeno es, en primer término, subjetivo y humano.

Palabras claves: desarrollo, comunidad, economía, antropología, capitalismo, mundialización.

Facultad de Economía, Universidad Autónoma de San Luis Potosí, México. Email: amaciasv@uaslp.mx

2 En la actualidad cursa estudios doctorales en la Universidad Complutense de Madrid. Email: quaitequeros@yahoo.com 


\section{Revista CUHSO volumen 8 nㅇ 1}

\section{ABSTRACT}

This work proposes a theoretical and conceptual reflextion about to development as an endogenous process. The viability of this perspective depends on, at a big extent, if its construction through the communitary life as subjetive space is assumed. The task is conplex but possible, because it seems to depend on the communities themselves and their capabilities to overcome their reflexive faculties to a retionalistic ethos proper from a civilization that progresively denies and disesteem its moral and affective dimensions. It is necessary, then, to overcome the determinist fatalism of the structural, the challenge of endogenous development is subjetive and human.

Key words: development, community, economy, anthropology, capitalism.

\section{INTRODUCCIÓN}

Toda economía es una forma particular e histórica de organización humana, ésta es una condición básica que nos dice que a lo económico lo social le es inherente. La constatación, aunque elemental, no deja de ser problemática, así al menos lo evidencian algunas sugerentes controversias que han configurado el campo de la antropología económica, especialmente desde la década de 1960; entre ellas, los debates que enfrentaron a sustantivistas y formalistas (Godelier 1976), a materialistas e idealistas (en Llobera 1981), hasta las más recientes elaboraciones sobre economías culturales (Bird-David 1997) y los enfoques críticos sobre la teoría del desarrollo.

En estos términos, hemos aprendido que la economía remite a un campo de relaciones subjetivas que, sin embargo, ha visto progresivamente socavado su sentido social en un trayecto particular en el cual prevalece y se expande, sin contrapesos, la racionalidad formal. Este es el punto de partida del formalismo, aquel que concibe a la economía como un hecho esencialmente abstracto y objetivo, basado en leyes que escapan a la voluntad humana; formalismo que, en la complejidad falaz de su proceso, tiende a predominar y a instituir al desarrollo como una de sus manifestaciones más verosímiles y conceptualmente más polémicas. Si bien partimos de la crítica, nuestro propósito es la búsqueda y la construcción de referentes teóricos que permitan imaginar el devenir de las comunidades humanas de un modo distinto al planteado por los enfoques estructurales predominantes en el pensamiento social y económico.

De la crítica al desarrollo subrayamos la vulnerabilidad conceptual de su aparente objetividad y su permanente adecuación como correlato de la teoría de la modernización (Domenach 1980). Teniendo en cuenta los sucesivos cuestionamientos a los enfoques desarrollistas -aquellos que identifican desarrollo y crecimiento económico- asumimos que lo relevante ya no es insistir en las connotaciones semánticas que uno u otro uso del término puedan reclamar; más relevante resulta profundizar e incluso ir más allá de las perspectivas que se plantean el desmantelamiento de su estatus ontológico. Como señala Arturo Escobar (1997) es necesario observar "las realidades locales desde sí mismas, 
independientemente de las constricciones conceptuales de un corpus teórico basado en la modernización capitalista".

Una perspectiva como la reseñada, fundada en el ámbito de lo subjetivo, nos aproxima a ciertas claves para reflexionar sobre el problema del desarrollo endógeno, por lo mismo no debe extrañar que se trate en primer término de una interpelación directa a la ciencia económica, en su dimensión práctica y teóricoepistemológica. En fin, no es posible abordar el problema del desarrollo -sea endógeno, inducido o adjetivado de cualquier otro modo- sin antes indagar en su fundamento: la economía.

El trabajo intenta sintetizar algunas constataciones respecto de la problemática relación entre comunidad, economía y desarrollo. En principio estas reflexiones, todavía en proceso de elaboración, tienen origen en seminarios de doctorado, en lecturas escogidas sobre economía, antropología económica y antropología política, en una investigación doctoral sobre comunidades costeras en la XI Región de Chile, y en permanentes debates sobre temáticas relacionadas. En resumen, el texto refleja un trabajo de orden teórico cuyo soporte ha sido la profundización en literatura especializada, no obstante, nuestra experiencia de investigación etnográfica y aplicada también puede considerarse reflejada en estos conceptos.

Pues bien, el texto aborda el problema del desarrollo endógeno desde una visión crítica y constructiva, intentando dar cuenta de sus concesiones, sujeciones y posibilidades. En primer término sintetizamos ciertos elementos que consideramos ineludibles, para introducirnos de forma relevante $y$ pertinente en el debate teórico contemporáneo, luego esbozamos una propuesta base en relación con la problemática del desarrollo, la economía y la comunidad, para finalmente ilustrar nuestro enfoque con algunas referencias al caso chileno.

\section{ECONOMÍA, DESARROLLO Y COMUNIDAD}

Se han escrito muchas páginas sobre los problemas teóricos y prácticos relacionados con el desarrollo económico. A la par, este concepto se ha ido calificando con sucesivos adjetivos, los cuales han supuesto una llamada de atención específica ante aquellos recursos y activos que el proceso de mercantilización y modernización económica dejaban a un lado, sin valorar su existencia. De este modo, se ha hablado de desarrollo sustentable, cultural, social, humano, etc., con el objetivo de que las estrategias y políticas de desarrollo económico atendiesen con especial énfasis los costes sociales y ambientales que el desarrollo capitalista ocasionaba en los territorios y comunidades que lo experimentaban.

La lógica con la que se ha tendido a enfrentar este tipo de externalidades negativas ha sido la de poner de manifiesto la potencialidad que estos activos poseen desde el punto de vista del mismo proceso de mercantilización y modernización. Se habla frecuentemente de la "puesta en valor" del patrimonio natural y/o cultural, como una manera de que este tipo de recursos, anteriormente desahuciados, sean valorados por medio de un precio y tengan la posibilidad de contribuir al proceso de desarrollo, impulsándolo en lugar de obstaculizarlo. En este sentido, la experiencia europea ha sido pionera en muchos de estos campos y ha abierto la posibilidad de imaginar genuinas estrategias de desarrollo para territorios y comunidades que previamente no disponían de ninguna ventaja competitiva, más allá de insertarse en los mercados como meros suministradores de materias primas y mano de obra barata ${ }^{3}$.

Sin embargo, no podemos asegurar que la aplicación de este tipo de estrategias de desarrollo haya sido exitosa en todos los casos, ni siquiera se ha podido evitar que la presión del mercado, en estos tiempos de globalización, haya deformado las intenciones primigenias de las sociedades civiles y de las administraciones estatales y regionales. Las cosas parecen ser más complicadas y a menudo resurge el desánimo y reaparece la siempre paralizante "cultura del subsidio", que fortalece la tendencia a renunciar a la necesaria auto-responsabilidad e inhibe los impulsos endógenos hacia la mejora de la vida material en un determinado contexto humano y territorial. Precisamente, en el caso de las regiones periféricas europeas, nos encontramos ante realidades donde

\footnotetext{
3 Lo interesante en las estrategias europeas ha sido también la metodología de trabajo, incorporando el partenariado como elemento implícitamente necesario para la aplicación de las políticas de desarrollo, logrando implicar desde un comienzo a los agentes locales no sólo en la toma de decisiones, sino en el propio análisis de las fortalezas y debilidades que los territorios poseen.
} 
aparecen entremezcladas ambas dinámicas de desarrollo, la endógena y la asistida, siendo difícil distinguirlas incluso para los investigadores y los agentes dinamizadores que externamente estudian e inciden sobre dichos contextos sociales.

La gran preocupación de los agentes de desarrollo local, de los investigadores del desarrollo territorial, ha sido incorporar en la dinámica interna de las comunidades un cierto comportamiento económico, capaz de hacer frente a la creciente competitividad en los mercados. Este ha sido el aspecto distintivo habitual de los enfoques de desarrollo endógeno: evitar que el proceso de desarrollo sea concebido simplemente con el resultado de una acción externa, normalmente basada en la aportación de recursos financieros, y comenzar a pensarlo como un proyecto comunitario, asumido subjetivamente y con mayor protagonismo por parte de los agrupamientos sociales. No se trata sólo de que el desarrollo se combine con la participación democrática, sino que realmente el centro, el origen del mismo sea la propia comunidad.

Esta intención en sí misma representa un importante punto de ruptura con las teorías del desarrollo económico surgidas con posterioridad a la Segunda Guerra Mundial, en el "caldo de cultivo" del Plan Marshall; pero desde el punto de vista de una construcción teórica alternativa sigue mostrando aspectos oscuros, especialmente en su dimensión subjetiva. En realidad, el comportamiento económico subjetivo que buscan los defensores del desarrollo endógeno no difiere del promulgado por la economía ortodoxa y los organismos económicos internacionales, aunque se diferencia y mucho en las palancas institucionales y sociales que activarían dicho comportamiento, necesariamente caracterizado por la eficiencia y el cálculo maximizador. Si la economía neoclásica y los organismos financieros internacionales defienden la apertura comercial y las reformas económicas de corte liberal como incentivo a la eficiencia y el comportamiento maximizador de utilidades, los defensores de enfoques neoinstitucionales, regulacionistas o del capital social no entienden que este tipo de incentivos se transmitan de forma tan automática, incidiendo particularmente en esa "caja negra" que son las características institucionales y organizativas de los entornos sociales donde se producen dichas transformaciones económicas. Ciertamente, en el origen de estas diferencias teóricas y de política, existen motivaciones éticas y morales que es necesario tomar en consideración, pero también es evidente que dentro de la relación entre medios y fines, siempre el desarrollo es el fin y la comunidad el instrumento.

En esta lógica, el desarrollo endógeno en realidad no lo es, no deja de ser un proceso donde permanente y recurrentemente la comunidad sufre una alineación de sí misma. El desarrollo aparece como una meta, que exige la transformación subjetiva de la comunidad, separándola de las cualidades humanas que histórica y culturalmente la han configurado como tal. Transformándose en microempresarios, por ejemplo, los miembros de una comunidad no parten de sí mismos para desarrollarse o al menos no parten de los fundamentos positivos que han posibilitado la construcción social de la misma.

Desde nuestro punto de vista, construir una estrategia endógena del desarrollo económico requiere partir de la comunidad en su integridad, aun más explícito: que la propia comunidad parta de sí misma, primando las facultades subjetivas que la fortalecen en detrimento de las que la debilitan, y que precisamente pueden ser incentivadas por la expansión del mercado si éste es concebido exclusivamente como parte de un mecanismo automático, caracterizado por leyes económicas objetivas sobre las cuales hay que "cabalgar" para intentar ganar la carrera de la competencia mercantil. No se trata de utilizar parámetros conservadores y "esencialistas" en referencia a lo comunitario, sino de medirnos con las motivaciones profundas que impulsan la cooperación y la conformación de una vida material organizada en comunidades, motivaciones que todavía pueden ser pensadas e imaginadas como factores subjetivos de auto-transformación social.

Por supuesto, esto exige empezar a elaborar un nuevo enfoque de la ciencia económica y de las ciencias sociales en general, que rompa radicalmente con la trayectoria y la hegemonía de la economía política, incluyendo las elaboraciones teóricas de sus críticos, en primer lugar de Karl Marx. Estamos empeñados en plantear una visión de la economía que sea verdaderamente subjetiva, porque es en primer término humanista. Un enfoque donde lo económico es concebido como una creación cultural humana, 
relacionada con la organización de la vida material de la especie (Macías, Bachiller y Saavedra 2004). En este sentido, el debate sobre el desarrollo endógeno, desde dentro, desde la comunidad, no puede ir desligado de un análisis de la dimensión históricoantropológica de los agrupamientos humanos que en sus relaciones sociales crean instituciones económicas.

Este análisis debe de construir una metodología innovadora, que rompa con las determinaciones objetivas de la modernidad. Es decir, las hipótesis de investigación deben de cambiar de dirección y superar esa concepción tan estrecha de las relaciones causaefecto. Por un lado, antes de explicar cómo afecta el mundo externo al mundo interno del individuo y las colectividades, se trataría justamente de invertir las variables que intervienen en las hipótesis: intentar analizar cómo el mundo interior repercute sobre la objetividad exterior, esto es, empezar a comprender el mundo como resultado de una elección humana, que, por otro lado, no está determinada, no obedece a causas que no permiten elegir. El ser humano no es prisionero de determinaciones estructurales, en primer lugar porque la evolución histórica nos demuestra que contextos semejantes han dado lugar a reacciones diversas por parte de las colectividades humanas. Tener esto presente no implica subvalorar las famosas condiciones objetivas, ni obviar los condicionantes estructurales que limitan la acción comunitaria. La cuestión fundamental es saber de dónde partir, teniendo en cuenta además algo importante: que las condiciones objetivas pueden ser asimiladas subjetivamente de formas muy diferentes, y que por tanto los comportamientos económicos y sociales ofrecen una variabilidad que no debe ser minusvalorada.

Esto nos remite a un punto crucial: la exigencia es disciplinaria y no meramente técnica, el desarrollo no es en sí mismo; por sobre los casos puntuales que nos sirven de material ilustrativo, expresa siempre un campo más complejo y profundo. En este sentido, no se trata únicamente de una crítica políticoinstitucional, se trata especialmente de una crítica a la propia "comunidad", que sirva como aporte base a su incesante proceso de construcción social y cultural. La primera condición para construir un proceso de desarrollo endógeno auténtico consiste en que la comunidad asuma conscientemente que su futuro no está escrito de antemano por fuerzas extrañas a su voluntad, que antes de dejarse vencer por el fatalismo es fundamental asumir la capacidad humana de elección con diferentes posibilidades, que van de la regresión a la revolución. Las prácticas asistencialistas se basan justo en lo contrario: la comunidad desconoce su responsabilidad, se adapta a los condicionamientos estructurales asociados al proceso de globalización, pero por ello no deja de realizar una elección subjetiva.

\section{LA MUNDIALIZACIÓN COMO CIVILIZACIÓN MATERIAL PECULIAR}

Tal vez, sea necesario hablar con más claridad: las teorías económicas de la modernidad han sido elaboradas a partir de un cierto tipo de civilización material, edificada a partir del siglo XVI en Europa Occidental. Se trata de enfoques teóricos totalitarios, no sólo en el sentido de que consideran que una lectura económico-política construida desde Occidente es suficiente para comprender las realidades sociales a escala planetaria, sino sobre todo $-y$ esto es realmente lo preocupante, pues entre otros motivos sigue prevaleciendo en las teorías postmodernasporque son visiones teóricas incapaces fatalmente de ser auto-conscientes y auto-reflexivas, son incapaces de tomar conciencia de lo que no conocen. Tomando un ejemplo muy pueril, pero que evidencia este tipo de lógica totalitaria de pensamiento, muchos economistas creen conocer la realidad económica de un país simplemente ojeando las grandes cifras macroeconómicas de los últimos años. Desde su posición prepotente, son capaces inmediatamente de emitir sentencias científicamente demostrables y de sancionar a las poblaciones con políticas económicas que en el caso de ser rechazadas por las mismas, lo serán-según tales personajes omniconscientes- porque ignoran realmente sus problemas.

El proceso que dio pie al surgimiento de esta civilización puede ser denominado como mundialización y, en nuestra opinión, posee entre otras las siguientes características sustanciales:

- Su formación, su consolidación y su crecimiento es incompatible con la pervivencia de 
otras formas de vida material, como se ha podido observar en la colonización americana y en el saqueo esclavista de África, pero también en la destrucción de la cultura material comunitaria en buena parte de Europa, como se pone de manifiesto en los trabajos sobre economía moral en Inglaterra del historiador social E.P. Thompson (1991: 213-293).

- La mundialización no sólo destruyó la cultura material de buena parte de las comunidades humanas del globo, sino que modificó sustancialmente la vida comunitaria, algo lógico si partimos de la premisa de que en estos agrupamientos sociales lo económico no aparece separado del conjunto de expresiones de la vida colectiva e individual.

- Esta transformación subjetiva a nivel comunitario fue especialmente intensa en Europa Occidental, preparando el terreno para el apogeo del Estado moderno como nuevo articulador de la vida material, fenómeno que va inseparablemente unido al propio proceso de mundialización.

- En aquellos lugares donde la comunidad resistió o donde el Estado fue enormemente débil para articular y organizar de forma hegemónica la vida material de las sociedades, la mundialización se manifestó en sus formas más íntimamente destructivas, tanto de recursos materiales como de seres humanos.

Efectivamente, como plantea Schumpeter (1942: 118-124), el capitalismo como civilización material puede ser enormemente creativo, constructivo, pero a condición de que sea asumido conscientemente en el comportamiento cotidiano de los sujetos económicos, comenzando por la figura del empresario, y no sólo. Si no es así, el capitalismo y el proceso de mundialización que ha ido aparejado a su expansión pueden ser dramáticamente destructivos no sólo de otras formas de vida material, sino de los propios seres humanos. Realmente, como decimos, esta faceta es la que ha prevalecido, y posiblemente esta característica se explica no por la existencia de unas ciertas leyes de la acumulación, que determinan la evolución de la sociedad y que periódicamente se manifiestan en crisis y destrucción del aparato productivo; sino porque la interiorización del comportamiento maximizador de las utilidades y el espíritu acumulador de las mismas se basa seguramente en ciertas facultades negativas de la especie humana, que ponen en peligro y cuestionan los fundamentos de su vida comunitaria, lo cual paradójicamente es puesto en evidencia por Adam Smith (1759: 185-186).

El propio Max Weber, a partir de su estudio sobre la influencia de la ética protestante en el surgimiento del capitalismo, no deja de poner de relieve este principio subjetivo que caracteriza todo funcionamiento económico. A contracorriente de las ideas dominantes en su época y de la propia economía política, y al margen de la lectura reduccionista que el funcionalismo realizó de su propia obra, este autor no puede concebir al ser humano como parte de un mecanismo automático caracterizado por leyes económicas objetivas, ajenas a su capacidad de elección. Para Weber, la forma capitalista de una economía y el espíritu con el que se la dirige no se relacionan en función de una ley de dependencia (Weber 1904-5: 73). Al igual que Schumpeter (1942: 68), el cual sostiene que el proceso social racionaliza nuestra vida y nuestro pensamiento alejándonos de un tratamiento metafísico del desarrollo social, previo a una interpretación científica del mismo; el pensador alemán plantea que el modo de vida racional constitutivo del capitalismo nació del espíritu del ascetismo cristiano (Weber 1904-5: 232).

Ciertamente, Weber hace referencia a un proceso de racionalización, que se caracteriza entre otros aspectos por la generalización de los comportamientos y su conversión en prácticas rutinarias. Más adelante, el sociólogo francés Pierre Bourdieu, en un recorrido con características propias, sostendrá que el predominio del hábito y de la costumbre sobre cualquier otra facultad humana hace que la subjetividad se reduzca a su dimensión práctica y que la institucionalización de los comportamientos se eleve a la categoría de propiedades funcionales de un sistema social (Bourdieu 2000: 13-28). Pero en cierto sentido este planteamiento teórico y metodológico resulta claramente insuficiente, especialmente desde el punto de vista de la construcción del conocimiento de las realidades sociales. De hecho, el pensamiento científico moderno ha basado el desarrollo de sus categorías analíticas precisamente tomando como referencia los comportamientos más mecánicos del ser humano, otorgándoles el carácter de leyes objetivas, revistiéndoles de un concepto alienado de cultura y 
en realidad dejando al margen las facultades reflexivas, no entendidas exclusivamente como simbólicas, basadas en la conciencia humana. En relación a esta cuestión tan consustancial al surgimiento de la modernidad, es interesante reproducir un breve extracto de un trabajo del fundador del institucionalismo norteamericano:

“La preconcepción de la realidad, por otra parte, refuerza una manipulación de los hechos sin imputación de fuerza o atención personal, pero con una imputación de continuidad mecánica, que es sustancialmente la preconcepción que ha alcanzado una formulación en manos de los científicos, bajo el nombre de conservación de la energía o persistencia de la cantidad. Cierto recurso apreciable a este último método de conocimiento es inevitable en cualquier estadio cultural, porque es indispensable para toda eficiencia industrial. Todos los procesos tecnológicos y todos los artilugios mecánicos descansan, psicológicamente hablando, sobre este fundamento. Este hábito de pensamiento es una consecuencia selectivamente necesaria de la vida industrial y, desde luego, de toda experiencia humana en el empleo de los medios de vida materiales. Debe seguirse por tanto, por lo general, que cuanto más elevada sea la cultura mayor es la proporción de preconcepción mecánica que conforma el pensamiento y el conocimiento humano, puesto que, por lo general, el estadio cultural al que se ha llegado depende de la eficiencia de la industria" (Veblen 1899b: 141) ${ }^{4}$.

Profundizando en las implicaciones de este debate, resulta interesante detenerse a analizar cómo enfrenta metodológicamente Thorstein Veblen el estudio de las instituciones económicas. Según este autor, la consolidación de unos hábitos de vida depredadores en la comunidad no son ajenos a una determinada característica de la subjetividad humana: la búsqueda en cada acción de la realización de un determinado fin concreto, objetivo e impersonal, que le otorgue prestigio ante sus semejantes. La aparición de esta característica sería el resultado de una transformación espiritual en el ser humano, pues en las comunidades primitivas no depredadoras no existe una antítesis entre el individuo y el resto de la realidad. Es más, el desarrollo y la diferenciación de las clases sociales

4 Citado en R.B. EKELUND y R.F. HÉBERT, Historia de la teoría económica y de su método. McGraw Hill, Madrid, 1997, pp. 484-485. no pueden ser explicadas al margen de conceptos como la dignidad, el valor y el honor, que se desprende de la destrucción de los competidores y la subordinación de las fuerzas extrañas que amenazan la pervivencia de la comunidad (Veblen 1899a: 1823). En esta lógica, la emulación sería una motivación humana que influye en el comportamiento económico al igual que otras motivaciones, como la necesidad o la capacidad de trascender.

\section{Repensar LA CULTURA MATERIAL DE LAS COMUNIDADES}

La cultura material de la modernidad ha sido enormemente destructiva: continentes enteros que han construido culturas materiales que les han permitido reproducirse durante siglos y milenios, recientemente sufren deterioros y crisis que ponen en peligro su propia subsistencia. Realmente, la agresividad de esta cultura está poniendo en cuestión la vida humana a escala planetaria, sin que parezca que nada ni nadie pueda frenarla.

En más de algún sentido, esta devastadora organización de la vida material predominante en Occidente es culturalmente impugnable en relación con la de aquellos pueblos denominados "primitivos", incluso en el mundo contemporáneo. Primero, porque estas comunidades han concebido la vida material hasta cierto punto como un ámbito de cooperación y de construcción de relaciones humanas solidarias, lo cual en nuestra sociedad moderna es inconcebible. Segundo, porque estas comunidades han desarrollado en mayor medida su capacidad de elección, la cual no está sujeta a leyes objetivas extrañas a sus criterios morales y sociales (Mauss 1924). Frente a nuestro consumismo enloquecido, frente a la presencia actual del hambre en economías exportadoras de alimentos, efectivamente las comunidades originarias tendrían mucho que enseñarnos, pues éstas elegían sus necesidades materiales, delimitando la jornada laboral en función de las mismas, y la elección de los medios era coherente con los fines no sólo en un sentido económico, sino social y moral (Sahlins 1972: 22-27). Seguramente, a estos seres humanos "primitivos", que no eran muy diferentes a nosotros ni racional ni sentimentalmente, les hubiera sido bastante difícil de justificar pagar una deuda con los alimentos cotidianos. 
Es evidente que en un mundo altamente dinámico (Clifford 1997) e interdependiente (Wolf 1987, Giddens 1997), salvo improbables excepciones, es cada vez más difícil y cuestionable referir la existencia de comunidades prístinas, aisladas o "inmunes" a las influencias de otras culturas y contextos sociales, dominantes o no. En este sentido, no se pueden minimizar los estragos que ha provocado el avance del capitalismo en todos los rincones del planeta; no obstante, tampoco es posible sostener que las comunidades rurales, campesinas e indígenas constituyen entidades pasivas, en proceso de descomposición e irremediablemente sujetas al devenir de la modernización. Como señalan los enfoques de la hibridación cultural (García Canclini 1990) estos colectivos tienen, en su dinamismo identitario, un innegable potencial de resignificación y replanteamiento de los procesos sociales y económicos que les afectan con mayor o menor profundidad. Desde este punto de vista, es justamente en dicho potencial, y a partir de éste, donde debemos comenzar la búsqueda de aquellos elementos que permitirían una construcción de lo social fundada en valores humanistas. Sin embargo, tampoco se trata de obviar lo evidente: es probable que la tarea sea más compleja y diversa, pues las tensiones, en los cada vez más ambiguos límites culturales, pueden hacer de aquella potencialidad una romántica ilusión de la antropología.

Pues bien, aun teniendo en cuenta estas limitaciones, es posible que, en y desde estos ámbitos culturales locales, sea viable plantear y encontrar las bases de proyectos alternativos al vendaval de devastación de la organización material predominante.

Todo depende de cómo enfoquemos la cuestión de la cultura, y más específicamente de la cultura material. Más allá de la polémica entre diferentes autores modernos y postmodernos en torno al concepto de cultura, nos interesa abordar esa tremenda paradoja relacionada con el hecho de que mientras la cultura llena nuestras vidas, sin embargo somos ampliamente inconscientes de ello (Herskovits 1948: 33). ¿Sería posible transformar las culturas en el sentido de dotarlas de un carácter auto-reflexivo y autoemancipatorio? Posiblemente sí, siempre y cuando superemos el determinismo cultural o, por el contrario, seamos capaces de romper con una visión de la cultura como remate simbólico del edificio social. Además, como condición suficiente, necesitamos abordar el desarrollo cultural y sus posibilidades futuras en su relación íntima con la conciencia humana. La formación de la conciencia representa uno de los aspectos más interesantes y todavía enigmáticos que se inserta en el nacimiento de la humanidad, en el contexto de las comunidades originarias fundadas sobre un instinto de solidaridad y cooperación (Veblen 1899a: 226). Desde entonces, el desarrollo de la conciencia humana ha sido escaso, traumático y sometido crecientemente a la presión del pensamiento racionalista. Pensar en un desarrollo endógeno, que permita a las comunidades enfrentar de una manera auto-propositiva y no sólo adaptativa los procesos de globalización, implica en primer lugar una batalla cultural en el sentido de educar en el fortalecimiento de las facultades concienciales que nos caracterizan como especie, significa en sus contenidos construir una cultura del bien común, que prepare un salto evolutivo a nivel de la conciencia, la más ignorada y descuidada de nuestras facultades humanas.

Si queremos construir un enfoque endógeno del desarrollo, debemos partir de la necesidad de fortalecer la capacidad de elección de las comunidades alrededor de la organización de la vida material. Creemos equivocado plantear el problema asumiendo, integrando, la subjetividad mercantil y egoísta que fundamenta el capitalismo, intentando asimilar a las personas en cuerpo y alma a la racionalidad económica que caracteriza la modernidad. No se trata tampoco de conservar las tradiciones, se trata de imaginar y configurar un camino propio de desarrollo, basándonos en las capacidades humanas, en el sentido planteado hipotéticamente por el economista y filósofo de origen indio Amartya Sen (1999: 19-28). Pero de alguna manera superando este enfoque, pues estas capacidades son en primer lugar concienciales y no determinadas unilateralmente por algún tipo de entramado institucional, como puede ser un sistema democrático.

La particularidad de esta "puesta en valor" y de su activación en un proceso de desarrollo exitoso está relacionada, entre otros factores, con la capacidad de la comunidad que habita un determinado territorio de tomar conciencia de la importancia de este objetivo 
asumiéndolo como aspiración colectiva, poniendo en práctica una estrategia organizativa que haga viable su incorporación al proceso de desarrollo, enriqueciéndolo. Max-Neef, en su enfoque sobre las necesidades humanas, identifica como recursos no convencionales la cultura organizativa y la capacidad de gestión, las redes sociales, la cualificación y la formación de los recursos humanos, la solidaridad y la capacidad de ayuda mutua. Lejos de agotarse con el uso, estos recursos presentes en algunos territorios se refuerzan, por lo que «la potenciación de recursos no convencionales estimula no sólo la autodependencia, sino que garantiza una mejor utilización de los recursos convencionales, especialmente del capital» (Max-Neef 1993).

El punto de partida es reflexivo porque demanda una capacidad "analítica" de lo cultural, como proceso dinámico y no estático, una capacidad de reflexión que permita a las comunidades, a los sujetos culturales, interactuar dialógicamente desde sí mismos, desde sus particularidades, con los procesos globales. Estas capacidades, al tener como referencia sus bases culturales, prácticas y conceptuales, admitirían evaluar en propiedad la posibilidad de transigir -y hasta qué punto- ciertos elementos en la ineludible construcción dialéctica de los proyectos colectivos. Siguiendo a Sahlins (1988), la subjetivación supone un proceso estructural de doble sentido, colectiva e individualmente: la cultura como realización de la estructura, o bien, la individualidad como riesgo estructural $y$, por tanto, como potencial de transformación cultural. Dicho de otro modo, el reto del desarrollo endógeno no es únicamente colectivo.

Nuestra concepción sobre el desarrollo parte entonces de la activación de ciertas capacidades comunitarias que en el fondo no son recursos objetivables, sino que hacen referencia a una concepción radicalmente subjetiva de la economía como creación cultural humana. Hay que dejar de tenerle tanto miedo al capitalismo y a su proceso de mundialización. La expansión capitalista no responde exclusivamente a fuerzas estructurales, determinadas objetivamente, sino que en primer lugar tiene una base humana: la aceptación y la asimilación subjetiva de sus valores y de sus prácticas. Esta base humana no se configura en una dimensión ideológica, sino que está estrechamente relacionada con nuestra naturaleza, con las características negativas que todos poseemos. En este sentido, la globalización capitalista no es una fuerza todopoderosa, algo inevitable que debemos sufrir, a lo que debemos de adaptarnos, y en el mejor de los casos asimilar en nuestras formas de vida para sacarle beneficio. Proponemos empezar a pensar los problemas de la vida material desde otra perspectiva, desde el punto de vista de nuestras características positivas de solidaridad y cooperación, superando los límites que han manifestado en los albores de la humanidad $^{5}$. Si el capitalismo en su mundialización, apoyado por las formas de organización estatal, ha sido capaz de destruir las formas comunitarias de vida material se explica en primer lugar por la debilidad conciencial de las colectividades humanas, que han "preferido" basarse en el hábito y en la costumbre, antes que en sus facultades reflexivas. La expansión destructiva, totalitaria, del capitalismo no es el producto inevitable de leyes económicas objetivas, que funcionan independientemente de las capacidades mentales y sentimentales de los seres humanos: ésta es una de las grandes falacias de la economía política.

La humanidad no ha dejado de proponer caminos alternativos, más o menos conscientemente. $Y$ de alguna manera, subterráneamente, muchas comunidades siguen haciendo vivir su espíritu cooperativo y solidario, antes que nada por su necesidad de vivir y sentir una dimensión afectiva más de conjunto. Esta fuerza humana, que hay que pensarla más y desarrollarla más allá de sus límites históricos y culturales, es seguramente una de las claves de un futuro posible.

\section{Desarrollo y comunidades RURALES EN ChILE}

Especialmente a partir de 1990, cuando Chile recupera su democracia política, emergen y se hacen visibles una serie de actores e instituciones orientadas a generar e intervenir en procesos de desarrollo. En el plano social-cultural, también cobran visibilidad aquellas subjetividades a las que en teoría están

5 Estos límites han consistido, básicamente, en cómo las relaciones de parentesco han dificultado el desarrollo de los ámbitos de cooperación, aunque de todas maneras es interesante analizar más detenidamente los instintos humanos que están detrás de determinadas formas de intercambio entre diferentes comunidades originarias. 
dirigidas dichas iniciativas y políticas. Se habla de comunidades campesinas, de pueblos indígenas, de pescadores artesanales; se habla de pobres, de superación de la pobreza, de desarrollo comunitario, de desarrollo social, de desarrollo económico y territorial. En cierto modo se configuraba un "campo del desarrollo", un campo que, por lo demás, es problemático en su relación institucional-social subjetiva. Por una parte nos encontramos con las instituciones y los agentes promotores del desarrollo $y$, por otra, con las comunidades, con los "sujetos" que se dice son los "beneficiarios" de las políticas y programas impulsados por los primeros. La relación se ha caracterizado por una asimetría básica en la que desde el nivel experto institucional, especialmente estatal, se intenta imponer a las comunidades una lógica y un tipo de desarrollo fundado en los paradigmas dominantes de la economía ortodoxa. Un modelo que, según hemos aludido, se enmascara en diversos formatos, pero que no admite cuestionamientos a la supuesta esencia objetiva de la razón maximizadora.

En los espacios rurales el despliegue de esta lógica y sus procedimientos es tan patente como inagotable. ¿Qué entienden los técnicos y los agentes del desarrollo como tal? Fundamentalmente microempresas, comercialización y competitividad individual ("intégrese al club de los emprendedores"). Pero resulta que este tipo de fórmulas no representan más que una solución intencionada entre muchas posibles, entonces ¿por qué no cualquier otra forma organizacional que permita la reproducción material de la comunidad? No cabe una respuesta más ingenua que la pregunta.

Todas las instituciones, organizaciones, agencias estatales o no estatales, que ponen al desarrollo como misión y objetivo, apuestan a dinamizar las economías locales. Hemos visto como punto crítico que esa noción de economía es totalitaria, noción que ciertamente predomina en las universidades y que antes nos habían "enseñado" en la educación secundaria. Noción que ha visto una de sus "épocas doradas" durante el impulso del neoliberalismo, cuyo laboratorio en el Tercer Mundo fue Chile en las décadas de 1970 y 1980; en esa época las carreras de Economía en las universidades chilenas cambian su nombre por el de "Ingeniería Comercial" y, de paso, se transforman en las escuelas del dogma de un solo tipo de economía, la misma que Max-Neef ha denominado la "economía patológica". Aquella que al ser incapaz de ver y aceptar a esas otras formas de reproducción de la vida material, niega, por ejemplo, a las economías campesinas o indígenas, y se propone -a cómo dé lugar- transformar a estos seres humanos en piezas articuladas funcionalmente al proyecto dominante o, en el peor y más frecuente de los casos, en excluidos del mismo.

Por supuesto que el ethos economicista, como podrá advertirse, no es atribuible a decisiones políticas de esta clase. Incluso la propia antropología económica no logra una ruptura sustancial con esta lógica sino hacia fines de los años ochenta, cuando surgen los enfoques de economías culturales (BirdDavid 1997).

De modo que, al estar inmersos en un pensamiento económico sesgado y prepotente (la falsa verdad de los cálculos matemáticos como método para someter toda realidad a mediciones), nos resulta muy difícil ver integralmente a esas otras formas de organización de la vida económica. Hemos sido socializados y educados de esa manera, fuimos condicionados (aunque no determinados) a ver la realidad de esa forma. Las diferencias entre las diversas intervenciones en desarrollo que tienen lugar en Chile no pasan por cuestiones de principios, pues en esencia son los mismos. Las personas que trabajan en estas instituciones han sido formadas, aleccionadas e instruidas, bajo una misma racionalidad económica.

Una de las características básicas de la economía de la maximización es la separación de los aspectos formales de los sociales. Lo social por un lado, lo económico por otro lado. Los economistas (Ilámense ingenieros y similares) a cargo de los cálculos, de la planificación, de la búsqueda de la fórmula adecuada, de la solución lógica; los sociólogos (y sus derivados) a cargo de lo social, algo entre folclórico y anecdótico, como si lo social fuese distinto de lo económico. No hay un área social y un área formal-racional en la organización económica de las comunidades, la confusión es absurda pero por desgracia es dominante. Un vistazo a los diseños de los distintos programas de desarrollo que operan en Chile y a sus procedimientos prácticos, es suficiente para constatar esta situación.

Es necesario abrir nuestras mentes "deformadas" (deformación reforzada en la academia) y ser capaces 
de aceptar, en primer lugar, y de ver, en segundo lugar, que existen otras lógicas económicas. No es posible que sigamos pensando que el desarrollo de una comunidad pasa por transformar a gran parte de sus habitantes en supuestos agentes empresariales, o que ese desarrollo llegará cuando esa comunidad ponga productos en los mercados, como si fuese un eslabón más del engranaje global o como si la única vía posible para las comunidades rurales fuese vender y mercantilizar sus recursos y patrimonios. Reducir las economías campesinas, las economías de los pueblos indígenas o de los pescadores artesanales del sur de Chile, a cálculos formales, a negocios, a comercialización, a emprendimientos individuales, no es otra cosa que contribuir a convertir al mundo campesino, indígena o costero en propiedad privada, en mercancía, en producto de compra y venta. Es esta la característica de las intervenciones en desarrollo de las instituciones públicas chilenas, bajo esta lógica operan, por ejemplo, los programas que promueve el Ministerio de Planificación, la Corporación de Fomento de la Producción, el Servicio de Cooperación Técnica, el Instituto de Desarrollo Agropecuario o el Fondo de Inversión Social y Solidaridad ${ }^{6}$ (por cierto, esta es también la lógica de sus continuos fracasos en los ámbitos rurales); pero también es la lógica prevaleciente en las organizaciones del "tercer sector" y particularmente en la cooperación internacional vinculada a los gobiernos del Primer Mundo ${ }^{7}$. De algún modo la razón práctica está sujeta al hábito ortodoxo, a la concepción formalista, instrumental u objetivista; y en este sentido no cabe demasiado optimismo, ¿acaso no sería disruptivo, e incluso amenazante para la lógica práctica de esta institucionalidad, una propuesta de desarrollo endógeno en los términos antes planteados?

Sin embargo, este condicionamiento deja de ser tan patente en todo un conjunto de instituciones y actores menos comprometidos con aquella identidad entre crecimiento económico y desarrollo. Así, en el

\footnotetext{
6 Estas tres últimas cuentan entre las más importantes oficinas a través de las cuales se materializan las políticas públicas de desarroIlo en Chile.

7 Por ejemplo la asistencia técnica de la Agencia de Cooperación Alemana, GTZ, en el plan de ordenamiento territorial en la XI Región, ha resultado especialmente consistente con los intereses expansivos de la industria productora de salmones en el borde costero de Aysén.
}

nivel institucional, es preciso que al menos ciertos organismos, por ejemplo los del "tercer sector", se permitan dudar de las verdades que se suponen universales, y establezcan aperturas hacia "nuevas" formas de entender la reproducción de la vida material de los pueblos. En esas formas, que más que nuevas nos son desconocidas, se encuentran importantes claves para construir un desarrollo, ya no completamente inducido desde la institucionalidad culta, sino para construir aquel tipo de desarrollo que hemos denominado endógeno; un desarrollo que, como hemos destacado, debe tener su punto de partida en las cualidades y capacidades culturales locales.

Frente a las acciones y a las decisiones institucionales, o frente las fuerzas devastadoras del mercado y de la modernización, las comunidades no están condenadas al inmovilismo. Si bien, como hemos referido, existen condicionamientos estructurales insoslayables, creemos que la dimensión subjetiva es potencialmente más relevante que lo sugerido por las concepciones deterministas.

Dos breves apuntes, sobre los conflictos del borde costero en el sur austral de Chile, nos servirán para ilustrar esta perspectiva:

En primer término una referencia a la tensión que produce la expansión de la industria salmonera. Sin entrar en detalles, señalemos que el proceso de privatización de las costas australes es cada vez más incesante, hecho que ha derivado en una proliferación desregulada y vertiginosa de centros de engorda en todo el archipiélago de Chiloé (años ochenta y noventa) y en el Archipiélago de los Chonos (desde mediados de los noventa hasta la fecha). De este modo, el borde costero comunal y con ello los sistemas tecnoeconómicos locales han visto progresivamente alterado su perfil ecológico y organizacional ${ }^{8}$.

La antropología define que la identidad, y ciertamente la cultura, es una construcción social

\footnotetext{
8 La expansión de los centros productores de salmón en las costas de la XI Región de Aysén, irá en constante aumento en los próximos cinco años, pues el objetivo de la industria es triplicar su producción en el decenio 2000-2010. Esto significará una enorme presión sobre los ecosistemas costeros, cuya magnitud podrá verse reflejada en la creciente saturación y contaminación del borde costero, en el deterioro medioambiental, en la desintegración social y la pobreza, y en la desestructuración de las economías locales.
} 
permanente. Sin embargo, esto no debe entenderse como un proceso "natural" que no pueda ni deba ser cuestionado o replanteado subjetivamente; Giddens refiere que las tradiciones son precisamente "medios de identidad", en tanto diferencian un "nosotros" de un hipotético "ellos", o un "los de dentro" de un "los otros" (1997: 103-111). Sostiene, además, que en la base de las tradiciones están las prácticas, unas más y otras menos vinculadas a saberes expertos, sujetos a lo que denomina los "guardianes" de la tradición. Señala Giddens, que en el caso de los Kung, del desierto del Kalahari, sus sistemas de caza y recolección se basan en un conocimiento práctico bastante especializado, el cual está evidentemente asociado a la experiencia acumulada de quienes ejercen la práctica de cazadores recolectores. De esto puede desprenderse que, para esta sociedad, el trabajo es un "depósito" de tradición, y por lo tanto un referente sustantivo de la identidad.

Aun teniendo en cuenta que existe una capacidad de transformación y resignificación en y desde las bases materiales de la comunidad, tampoco es menos cierto que es necesario que dichas capacidades se pongan en escena conscientemente, reflexivamente, desde la comunidad; de lo contrario, ante la implacable "embestida" del desarrollo, no tendrá lugar una transformación endógena de la tradición, sino su colapso, su descomposición cultural.

El razonamiento puede aplicarse perfectamente a las comunidades de pescadores artesanales del sur austral de Chile. En sus interacciones con la "avanzada del progreso", estas comunidades costeras no ponen en riesgo su identidad, ni su tradición; pues, tradición e identidad son categorías que en sí mismas se definen por estar en riesgo. Más relevante sería sostener que a estas comunidades se les presenta el desafío de redefinir las estrategias prácticas, concernientes a sus bases tecnoeconómicas. Así las cosas, las nuevas condiciones que impone la modernización les impelen también a transformar sus tradiciones de reproducción de la vida material. En este sentido, gran parte de su destino como cultura y como sociedad costera depende de sí mismas. Pues entonces, dada la expansión territorial de la industria del salmón y la especulación financiera que apareja, convendría que estas comunidades replanteasen, por ejemplo, su relación política y cotidiana con el espacio-borde costero. De no asumir como propia esta tarea, la vieja historia latinoamericana de usurpaciones territoriales volverá a recrearse; en cierta forma, se trata de que estas comunidades reinventen sus tradiciones y sus identidades económicas. A partir de aquí, el camino de aprendizaje cultural puede y debe ser interminable.

Como segunda cosa, y más allá de posibles idealizaciones sobre la vida comunitaria, digamos que la tarea es enormemente compleja. En el caso de los pueblos costeros y rurales de la XI Región de Aysén, tiende a prevalecer una lógica asistencial que les lleva a suponer que el Estado o algún ente benefactor les dará todo simplemente porque tiene la obligación de hacerlo; también, en algunos casos, existe un entramado de relaciones patronales de las cuales las comunidades parecieran estar presas, como si la gente tendiese a buscar un patrón que cumpla la función de organizarles parte de la vida. Este tipo de prácticas deviene cristalizadas como formas de ser aparentemente objetivas, pero que sabemos se han instituido a lo largo de historias particulares, y cuyo origen es posible y necesario desentrañar.

Es imprescindible que las comunidades superen estas y otras ataduras, que evidentemente son de tipo cultural y que muchas veces les dificultan tomar las riendas de su destino. Romper la lógica asistencial y la relación patronal es un primer paso que deben dar para librarse de sus limitaciones internas. Esto es fundamental para que la gente de Aysén, y probablemente de otras zonas rurales del país, sea capaz de asumir sus propios proyectos de desarrollo, o mejor, sus proyectos culturales, lo que hemos referido como desarrollo endógeno. ¿La condición primera? Lo destacábamos antes: unas facultades reflexivas que permitan a la subjetividad volverse libremente sobre sí misma.

\section{CONCLUSIONES}

Los cuatro siglos de modernidad han provocado un callejón sin salida en el pensamiento científico y no sólo también han proyectado una envolvente angustia vital, que hoy en día ya se presiente en todos los órdenes de la existencia. Decididamente, 
necesitamos ideas, cultura, para vivir mejor, no para racionalizar permanentemente nuestros sacrificios y nuestros límites. Necesitamos superar las hipótesis teóricas del pensamiento occidental, que finalmente son hipótesis de vida. Las relaciones sociales y económicas no están determinadas, sino que suponen una dimensión más de invención y de proyección. La tensión a la trascendencia puede desempeñar un mayor protagonismo, más allá del jugado en ese proceso de extrañación y de objetivación de las facultades sentimentales humanas que son las religiones y los mitos, que explican entre otros factores, ya mencionados anteriormente, los límites de sedimentación conciencial que se observan en las formas de cooperación originaria de las primeras comunidades humanas. Tenemos la oportunidad de partir de nuestra naturaleza facultativa y de nuestra conciencia, integrando aquello que ha sido separado por las tradiciones modernas y premodernas: la razón de los sentimientos, las necesidades materiales de las espirituales y afectivas, las ideas de las emociones, la tensión a la abstracción de la tensión a la trascendencia.

La economía no puede ser pensada independientemente de la capacidad humana para pensar y para proyectar hacia el futuro el bienestar integral de la especie. El verdadero sentido de la economía está atravesado por el entrelazarse de tres tensiones antropológicas: la necesidad, la aspiración y la trascendencia; lo cual aparece como exactamente opuesto a las lógicas mecánicas que las diferentes teorías económicas han desplegado a lo largo de la historia. La capacidad humana de invención económica se expresa precisamente en una diversidad de problemas y de soluciones que escapan, en el propio discurrir histórico, a toda visión economicista, etnocéntrica, progresista e iluminista. Es curioso observar cómo la ciencia económica ha sido y es una ideología de la escasez y a la vez se ha ido convirtiendo en la mecánica de una producción ilimitada, más marcadamente cuantitativa que cualitativa. De esta manera, se ha tratado de anular la posibilidad de pensar y de sentir lo que se necesita, lo que es suficiente, encerrando el espíritu humano en la lógica del productivismo y de la acumulación en beneficio de una minoría.

Un replanteamiento teórico del desarrollo endógeno debe tener presente esta condición de la lógica de la ciencia económica. Por otro lado, es conveniente que tome en consideración que el proceso de mundialización desde el siglo XVI no ha producido un desarrollo capitalista orgánico más allá de sus centros históricos. El problema no son las comunidades y su incapacidad para asumir un comportamiento capitalista, sino el propio desarrollo capitalista, incapaz de endogeneizar otro tipo de lógicas económicas y sociales.

Pensando en las relaciones cooperativas de las comunidades originarias hace miles de años, podríamos transformar las hipótesis, darles la vuelta, pero no sólo. ¿La tensión a la cooperación de nuestros ancestros era sólo la respuesta instintiva a las presiones de la supervivencia o, sin negar esta posibilidad, respondía a necesidades sentimentales como la necesidad de cercanía, de afecto, de amor o de serenidad? Podemos incluso preguntarnos por las necesidades materiales que acucian a la humanidad de hoy en día: ¿Qué necesitamos más: pan o amor? No es una pregunta banal. Tal vez, el pan pueda ser mejor, más sabroso y para todos si lo pensamos a partir de los sentimientos, desarrollando una razón sentimental.

Es más, se puede comenzar a plantear una hipótesis transgresora: sería posible mejorar las condiciones materiales de la vida humana si se desarrollasen las facultades sentimentales más positivas de la especie, que en su expresión actual demuestran que posiblemente los seres humanos no hemos superado una fase infantil. Esta hipótesis, que implica una actitud existencial, potencialmente supone un punto de partida para empezar a pensar una reinvención de la dimensión económica de la especie humana. Depende de todos nosotros, de la capacidad de razonar de una manera que no estamos acostumbrados, partiendo más conscientemente de lo que nos une y nos puede unir, del bien de cada uno y del bien común.

Ciertamente, existe otra manera de mirar el mundo y de mirarnos a nosotros mismos, más hoy que asistimos al agotamiento del pensamiento occidental, solapado en la prepotencia institucional y material. Este agotamiento refleja la incapacidad del poder occidental para interrogarse sobre los orígenes, sobre las raíces y las bases de existencia de la civilización de la cual se reclama. Es la consecuencia de la 
pretensión histórica de construir un saber y una lectura de la realidad unilateralmente en clave económicopolítica, a partir de la evolución histórica europea, incapaz de admitir su propia y absoluta ignorancia en relación a otras culturas y creencias terrenales. En realidad, la nube tóxica de la modernidad lo sigue envolviendo todo, condicionando las esperanzas y los placeres de la vida, reduciendo al ridículo los sentimientos más auténticos y más íntimos, convirtiendo en valores las dimensiones más mecánicas del hacer humano, sin excluir la violencia y la capacidad de hacer el mal.

\section{BIBLIOGRAFÍA}

BOURDIEU, P. (2000), Las estructuras sociales de la economía. Anagrama, Barcelona, 2003.

BIRD-DAVID, N. (1997), La economías: una perspectiva económico cultural. Revista Internacional de Ciencias Sociales 154, UNESCO, disponible en www.unesco.org/issj/rics154.html.

CLIFFORD, J. (1997), Itinerarios transculturales. Gedisa, Barcelona.

DOMENACH, J. M. (1980), Crisis del desarrollo, crisis de la racionalidad. En: El mito del desarrollo, por J. Attali, C, Castoriadis, J-M. Domenach, P. Massé, E. Morin y otros. Kairós, Barcelona, pp 13- 42.

ESCOBAR, A. (1997), Antropología y desarrollo. Revista Internacional de Ciencias Sociales 154, UNESCO, disponible en www.unesco.org/issj/ rics154.html.

GARCÍA CANCLINI, N. (1990), Culturas híbridas. Grijalbo, México.

GIDDENS, A. (1997) Vivir en una sociedad postradicional. En: Modernización Reflexiva, Beck, U., Giddens, A. \& Lash, S. Alianza Editorial, Madrid, pp. 75-136.

GODELIER, M. (1976), Antropología y economía. Anagrama, Barcelona.
HERSKOVITS, M. J. (1948), El hombre y sus obras. Fondo de Cultura Económica, México D. F., 2004.

LLOBERA, J. R. (1981), Antropología económica. Anagrama, Barcelona.

LUXEMBURGO, R. (1912), La acumulación de capital. Grijalbo, Barcelona, 1986.

MACÍAS, A.; BACHILLER, S. \& SAAVEDRA, G. (2004) Repensar la economía como cultura de la vida material. Economías 4, Cuenca, Ecuador.

MAUSS, M. (1924), Ensayo sobre el don. En Sociología y Antropología. Tecnos, Madrid, 1970.

MAX-NEEF, M. (1993), Desarrollo a escala humana. Conceptos, aplicaciones y algunas reflexiones. Icaria-Nordan Comunidad, Barcelona.

SAHLINS, M. (1972). Economía de la edad de piedra. Akal, Madrid, 1983.

(1988), Islas de historia: la muerte del capitán Cook, metáfora, antropología e historia. Gedisa, Barcelona.

SCHUMPETER, J. A. (1942), Teoría del desenvolvimiento económico. Fondo de Cultura Económica, México D.F., 1997.

SEN, A. (1999), Desarrollo y libertad. Planeta, Barcelona, 2000.

SMITH, A. (1759), Teoría de los sentimientos morales. Alianza Editorial, Madrid, 1997.

THOMPSON, E. P. (1991), Costumbres en común. Crítica, Barcelona, 1995.

VEBLEN, T. (1899a), Teoría de la clase ociosa. Fondo de Cultura Económica, Madrid, 2002.

WEBER, M. (1904-5), La ética protestante y el espíritu del capitalismo. Alianza Editorial, Madrid, 2001.

WOLF, E. (1987), Europa y la gente sin historia. Fondo de Cultura Económica, México, D.F. 